\title{
The role of IgM antiphenolic glycolipid-1 antibodies in assessing household contacts of leprosy patients in a low endemic area
}

\author{
D. J. SOARES, S. FAILBUS, Y. CHALISE \& \\ B. KATHET \\ Anandaban Leprosy Hospital, Post Box 151, Kathmandu, Nepal
}

Accepted for publication 27 May 1994

\begin{abstract}
Summary This study was carried out to assess the role PGL-1 antibodies may have to play in assisting with early diagnosis in close contacts of leprosy patients. Blood samples were collected from patients and contacts. It was found that $6 \cdot 9 \%$ of index cases and $1 \%$ of healthy contacts were positive for PGL-1 antibody. None of the healthy contacts developed clinical leprosy and all had become seronegative at follow-up. We conclude that screening for PGL-1 antibodies has a limited role in the screening of healthy contacts and may not be of use in low endemic areas.
\end{abstract}

\section{Introduction}

The effective implementation of multidrug therapy (MDT) for the treatment of leprosy in a geographically defined region results in the reduction in the number of leprosy patients and the case load for the leprosy control programme. Optimal strategies for active case detection in such low endemic areas where MDT has been implemented have yet to be defined. The relative risk for development of clinical leprosy is greater in the household contacts of leprosy patients than in the general community. ${ }^{1}$ There are, however, substantial differences in the rates of leprosy in close contacts of patients between different regions, with higher attack rates being associated with communities with a higher overall prevalence of leprosy. A number of cross-sectional serological studies have demonstrated an increased proportion of household contacts of leprosy patients with IgM antiphenolic glycolipid-1 (PGL) antibodies, than in the general community (reviewed by $\mathrm{Smith}^{2}$ ). In one large prospective study of the contacts of leprosy patients in a high endemic area, there was a strong association between the antiPGL-1 antibody levels and the risk of leprosy. ${ }^{3}$ Therefore, screening for antiPGL-1 antibodies in household contacts may help in detecting those at increased risk of disease.

Active leprosy control measures have been undertaken in the Lalitpur District in the Central Region of Nepal, close to Kathmandu, since 1962 when the Anandaban Leprosy Hospital was established. This was intensified in 1986 when a mass survey of the district was commenced. Active case-finding and the implementation of the World Health 
Organisation (WHO) recommended MDT regimens ${ }^{4}$ was conducted until 1990. During the period of the survey, 234 new cases were registered from a district population of 210,538 , a case-detection rate of $2 \cdot 2 / 10,000$ per year. For the purposes of this study, during 1991-92 all registered patients in the district were revisited and their household contacts examined. In order to assess the additional benefit of testing for antiPGL-1 antibodies in this low endemic area, blood samples were collected from both patients and contacts and tested for antiPGL-1 antibodies.

\section{Materials and methods}

During 1991-92 every patient ever registered in the Lalitpur District was visited at home by paramedical workers. The southern area of this district has poor access and therefore leprosy control staff only visit there occasionally. The current status and residence of patients were updated. Many patients had moved away from the district or died. All previous patients and those patients currently receiving MDT who were home at the time of the initial visit or one follow-up visit were examined physically with detailed voluntary muscle testing and sensory examination. Skin smears were performed on these patients and a finger prick blood sample was collected for antiPGL-1 antibodies. The healthy household contacts of these patients were also examined and where possible a capillary blood sample was also collected. Capillary blood samples were collected onto chromatography paper (Whatman, UK) which was stored with desiccant at $-20^{\circ} \mathrm{C}$ until tested. A 6-mm circle was punched out of the blood spot and eluted overnight in $500 \mu 1$ of $0.9 \mathrm{M}$ phosphate-buffered saline, ph $7 \cdot 2$, with $0.05 \%$ Tween 20 . The eluant, which was approximately a 1:50 dilution of serum, was then used in IgM antiPGL-1 enzyme linked immunosorbent assay (ELISA).

This utilized the glycoconjugate, disaccharide-bovine serum albumin (dBSA), provided through the Immunology of Leprosy Programme of WHO, in a solid-phase ELISA as previously described. ${ }^{5,6}$ Samples with an absorbent $>0 \cdot 199$, the mean +3 standard deviations of serum samples from 91 healthy Nepali subjects, were considered positive. Previously a strong correlation between simultaneous venous and capillary blood samples for antiPGL-1 antibodies was demonstrated in a group of 62 leprosy patients $\left.r_{8}=0.81, P<0.001\right)$ (data not shown).

Table 1. The classification of index cases and their contacts and the number in each group who were IgM antiPGL antibody positive

\begin{tabular}{|c|c|c|c|c|c|c|c|}
\hline Classification* & TT & BT & BB & BL & LL & IN & PN \\
\hline No. of index cases & 23 & 76 & 6 & 23 & 16 & 3 & 12 \\
\hline Total no. of contacts & 61 & 194 & 12 & 64 & 38 & 3 & 31 \\
\hline PGL-1 Ab + ve contacts & 0 & 2 & 1 & 0 & 0 & 0 & 1 \\
\hline PGL-1 Ab - ve contacts & 61 & 192 & 11 & 64 & 38 & 3 & 30 \\
\hline
\end{tabular}

\footnotetext{
*Classification: tuberculoid (TT), borderline-tuberculoid (BT), midborderline (BB), borderline lepromatous (BL), lepromatous (LL), indeterminant (IN) and primary neuritic (PN).
} 
Table 2. Year of release from treatment for the different types of index cases

\begin{tabular}{|c|c|c|c|c|c|c|c|}
\hline Classification & TT & BT & BB & BL & LL & IN & PN \\
\hline \multicolumn{8}{|l|}{ RFT year } \\
\hline 1970-79 & 1 & 2 & 1 & 1 & 0 & 0 & 0 \\
\hline $1980-84$ & 11 & 37 & 3 & 5 & 7 & 1 & 5 \\
\hline $1985-89$ & 8 & 22 & 0 & 5 & 5 & 2 & 4 \\
\hline 1990-92 & 2 & 11 & 1 & 8 & 1 & 0 & 0 \\
\hline On treatment & 1 & 4 & 1 & 4 & 3 & 0 & 3 \\
\hline
\end{tabular}

* Classification as in Table 1 .

\section{Results}

A total of 159 leprosy patients (113 male, 46 female) were re-examined. The classifications of these index cases, all of whom were adults, are shown in Table 1. The duration since release from chemotherapy varied from 1 to 22 years with a mean duration of 5 years (Table 2). In all, 16 of the index cases were still receiving chemotherapy. Of the index cases, 55 had been treated with multibacillary (MB) MDT, 93 with paucibacillary (PB) MDT and 11 had received dapsone monotherapy alone.

During this follow-up survey, 403 household contacts of 159 index cases were examined. Of these contacts, 178 were male, 225 female and 93 were aged $<15$ years. No new cases of leprosy were detected among the 403 contacts.

Of the index cases, $11(6.9 \%)$ had IgM antiPGL-1 antibodies. The proportion of index cases who were seropositive among those still receiving chemotherapy $(4 / 16,25 \%)$ or who had ceased chemotherapy in the preceding 2 years $(4 / 23,17 \cdot 4 \%)$ were higher (Table 3$) ; 4$ of the contacts $(1 \cdot 0 \%)$ were positive for IgM antiPGL antibodies (Table 4$)$. The index cases of the antibody positive contacts (2BT, 1BB, 1PN) were antibody negative at the time of testing (Table 1) and had completed therapy for variable periods of time (Table 3 ). None of the 4 antibody positive contacts had clinical features of leprosy. All 4 subjects were re-examined 6 months later when there were also no features of leprosy. On repeat testing, the antiPGL-1 antibodies had become negative at the 6-month follow-up.

Table 3. IgM antiPGL-1 antibodies in index cases and contacts analysed by year of release from treatment

\begin{tabular}{|c|c|c|c|c|}
\hline & \multicolumn{2}{|c|}{ Index case } & \multicolumn{2}{|c|}{ Contact } \\
\hline & $A b+v e$ & $A b-v e$ & $A b+v e$ & $\mathrm{Ab}-\mathrm{ve}$ \\
\hline \multicolumn{5}{|l|}{ RFT year } \\
\hline 1970-79 & 0 & 5 & 0 & 4 \\
\hline $1980-84$ & 3 & 66 & 2 & 181 \\
\hline $1985-89$ & 0 & 46 & 1 & 108 \\
\hline 1990-92 & 4 & 19 & 1 & 57 \\
\hline On treatment & 4 & 12 & 0 & 38 \\
\hline
\end{tabular}


Table 4. Actual absorbance values for antiPGL-1 antibody in the 4 healthy contacts (normal $<0 \cdot 200)$

\begin{tabular}{ccc}
\hline Contact & & \\
1 & 35-year-old female & $0 \cdot 276$ \\
2 & 8-year-old male & $0 \cdot 226$ \\
3 & 35-year-old female & $0 \cdot 959$ \\
4 & 21-year-old female & $0 \cdot 355$ \\
\hline
\end{tabular}

\section{Discussion}

The role of PGL-1 antibodies in the serodiagnosis of leprosy has been examined in a number of cross-sectional household studies. ${ }^{2}$ The seropositivity rate in household contacts ranged from 7 to $43 \%$ with higher rates being observed in contacts of MB cases. In contrast only $1.0 \%$ of the contact group in this study had demonstrable IgM antiPGL-1 antibodies. This may reflect the fact that $89 \%$ of the index cases had completed chemotherapy, in many cases up to 10 years beforehand. Further, the overall prevalence of registered leprosy case load had fallen dramatically in the district from $70 / 10,000$ to $2 \cdot 2 / 10,000$ over a 20 -year period (unpublished observations). Therefore the exposure to leprosy in the household and community was also substantially reduced.

As there were no new cases among this group of contacts examined no test can be used to define a high risk group. However, in a low endemic situation there are few new cases and those that occur do not seem to be related to close exposure to an index case. This is typical, however, for many low endemic districts following the successful implementation of leprosy control programmes.

The small number of seropositive subjects had no evidence of leprosy and rapidly became seronegative. Although there is an increased risk of leprosy in seropositive household contacts the majority of such individuals in follow-up studies in the Philippines (92\%), ${ }^{7}$ Tahiti $(99 \%)^{8}$ and Papua New Guinea (99\%), ${ }^{9}$ did not develop disease over 2-5 year follow-ups. Rather than being a marker of clinical leprosy, IgM antiPGL-1 antibodies may be evidence of subclinical infection, particularly in children and adolescents ${ }^{9,10}$ which revert to negative spontaneously. Further, in longitudinal studies both in household contacts ${ }^{3}$ and in the general population, ${ }^{9}$ the majority of new leprosy cases develop in seronegative rather than seropositive subjects. Therefore screening for IgM and antiPGL-1 antibodies has a limited role in the screening of contacts of leprosy patients in a low endemic region.

\section{Acknowledgments}

We thank the leprosy control staff at Anandaban Leprosy Hospital for their assistance in collecting the blood samples. We are grateful to Dr W. J. Britton, Senior Lecturer in Immunology, Department of Medicine, University of Sydney for his many helpful suggestions and comments. 


\section{References}

${ }^{1}$ Nordeen SK. The epidemiology of leprosy. In: Hastings RC, ed. Leprosy. Edinburgh: Churchill Livingstone, 1985: 15-30.

${ }^{2}$ Smith PG. Serodiagnosis of leprosy. Lepr Rev, 1992; 63: 97-100.

${ }^{3}$ Ulrich M, Smith PG, Zuniga M, et al. IgM antibodies to native phenolic glycolipid I in contacts of leprosy patients in Venezuela: epidemiological observations and a prospective study of the risk of leprosy. Int $J$ Lepr, 1991; 59: 405-15.

${ }^{4}$ WHO Study Group. Chemotherapy of leprosy for control programmes. Technical Report Series 675. 1982. World Health Organization, Geneva.

${ }^{5}$ Brett SJ, Payne SN, Gigg J, Burgess P, Gigg R. Use of synthetic glycoconjugates containing the M. leprae specific and immunodominant epitope of phenolic glycolipid I in the serology of leprosy. Clin exp Immunol, 1986; 64: 476-83.

${ }^{6}$ Roche PW, Britton WJ, Failbus SS. Williams D, Pradhan TM, Theuvenet WJ. Operational value of serological measurements in multibacillary leprosy patients: clinical and bacteriological correlates of antibody responses. Int J Lepr, 1990; 58: 480-90.

${ }^{7}$ Douglas JT, Celona RV, Abalos RM, Madarang MG, Fajardo T. Serological reactivity and early detection of leprosy among contacts of lepromatous leprosy patients in Cebu, the Philippines. Int J Lepr, 1987; 55: $718-21$.

${ }^{8}$ Chateau S, Cartel JL, Guidi C, Plichart R, Bach AM. Seroepidemiological study of 724 household contacts of leprosy patients in French Polynesia using disaccharide-octyle-BSA as antigen. Int J Lepr, 1987; 55: $626-32$.

9 Baumgart KW, Britton WJ, Mullins RJ, Basten A, Barnetson RSC. Subclinical infection with M. leprae: a problem for leprosy control strategies. Trans Roy Soc Trop Med, 1993; 87: 412-15.

10 Baumgart KW, Britton WJ, Basten A, Bagshawe A. Use of phenolic glycolipid 1 for serodiagnosis of leprosy in a high prevalence village in Papua New Guinea. Trans Roy Soc Trop Med, 1987; 81: 1030-2. 